\title{
Ciprofloxacin eye drops-induced subtherapeutic serum phenytoin levels resulting in breakthrough seizures
}

\author{
Srinivasa Sastry Malladi ${ }^{1}$, MBBS, MRCPsych, Emily Kai Suen Liew ${ }^{1}$, BSc, Xiao Ting $\underline{N g}^{1}$, mB BCh BAO, Rita Kheng Siew $\underline{\text { Tan }^{1}}$, mN, BHSc
}

\begin{abstract}
An 81-year-old woman with a history of temporal lobe epilepsy-induced psychotic episodes was initially admitted to a general hospital where she was started on a course of oral antibiotics for community-acquired pneumonia, and ciprofloxacin eye drops to treat nasolacrimal duct obstruction. After one week, the patient was discharged back to a nursing home with these medications. However, she was admitted to our psychiatric ward two days later due to a relapse of psychosis. Another six days later, she developed breakthrough seizures associated with subtherapeutic serum phenytoin levels. Having explored all possible causes of reduced serum phenytoin levels, ciprofloxacin eye drops was discontinued in the patient, resulting in gradual return of phenytoin levels to the therapeutic range, with no further seizures observed in the patient.
\end{abstract}

Keywords: ciprofloxacin, phenytoin, seizures

\section{INTRODUCTION}

Although interaction between drugs administered topically and those administered orally is possible, clinical manifestations may not be commonly observed. Herein, we report a case of such a drug interaction between ciprofloxacin eye drops and oral phenytoin, which led to breakthrough seizures in an elderly woman with a known history of temporal lobe epilepsy and secondary psychotic episodes. Having excluded other possible causes of breakthrough seizures in our patient, we concluded that ophthalmic ciprofloxacin was most likely to have caused the marked reduction of serum phenytoin to subtherapeutic levels, leading to breakthrough seizures in the patient.

\section{CASE REPORT}

An 81-year-old Chinese woman with a more than 25-year history of temporal lobe epilepsy and secondary psychotic episodes was initially admitted to a general hospital for general functional decline. There, she was treated for community-acquired pneumonia with oral Augmentin $625 \mathrm{mg}$ (GlaxoSmithKline, London, UK) three times a day and clarithromycin $500 \mathrm{mg}$ twice a day for one week. Ciprofloxacin $0.3 \%$ eye drops was prescribed for two weeks to treat nasolacrimal duct obstruction of the left eye, at a dose of one drop in each eye, four times a day. On the day of admission to the general hospital, the patient's serum phenytoin level was $20 \mathrm{mg} / \mathrm{L}$. She was discharged back to her nursing home after a week-long inpatient stay.

However, two days later, she was admitted to our psychiatric ward for a relapse of psychosis. She was otherwise medically well with no recent seizure activity. Her medical history included seborrhoeic dermatitis, previous cerebrovascular episodes with pseudobulbar palsy, gallstones with transaminitis, alpha thalassaemia trait and clumped thrombocytopenia. Her medications on admission to our psychiatric ward were oral Augmentin, calcium carbonate, clarithromycin, clonazepam, folic acid, lactulose, Neuroforte ${ }^{\circledR}$, phenytoin, risperidone, and topical ciprofloxacin $0.3 \%$ eye drops, Tears Naturale II eye drops and an aqueous cream. The patient's psychotic symptoms resolved after a few days, with no significant changes to her antipsychotic medication. While arrangements were being made to discharge her back to the nursing home, she started experiencing breakthrough seizures. Her serum phenytoin level measured the following day was found to have dropped to $2 \mathrm{mg} / \mathrm{L}$. Apart from a mild elevation of gamma-glutamyl transferase, the patient's liver function test was within normal limits. Over the next two days, she experienced a few more episodes of seizures. Following the onset of seizures, the serum phenytoin level dropped further to $1 \mathrm{mg} / \mathrm{L}$ on Day 4.

The ward nurses confirmed that the patient was compliant with her medications. Therefore, possible drug interactions were explored with the help of a pharmacist. Oral antibiotics were discontinued and the dosage of phenytoin at night was increased from $300 \mathrm{mg}$ to $330 \mathrm{mg}$. On Day 7, the patient's serum phenytoin level still remained at less than $2 \mathrm{mg} / \mathrm{L}$. After ruling out all other possible reasons for the decrease in serum phenytoin levels in the patient, ciprofloxacin eye drops was discontinued. Serum phenytoin level gradually rose to $11 \mathrm{mg} / \mathrm{L}$ by Day 12 and $15 \mathrm{mg} / \mathrm{L}$ by Day 16 , the fifth and ninth day after the discontinuation of ciprofloxacin eye drops, respectively. In view of the patient's previous history of phenytoin toxicity, the phenytoin dose was reverted to $300 \mathrm{mg}$ at night. As the patient remained seizure-free, she was discharged back to the nursing home. At subsequent follow-up at two weeks, the patient's serum phenytoin level had returned to the baseline level of $20 \mathrm{mg} / \mathrm{L}$.

\section{DISCUSSION}

Systemic absorption of ciprofloxacin is possible after the administration of topical ocular medication, with plasma levels in human subjects ranging from nonquantifiable to $4.7 \mathrm{ng} / \mathrm{mL}$, 
which is approximately 450-fold less than that seen following a single oral dose of $250 \mathrm{mg}$ ciprofloxacin. ${ }^{(1)}$ However, information on any specific drug interaction studies conducted with ophthalmic ciprofloxacin is not available. Quinolones have been known to either trigger seizures or lower the seizure threshold. Ciprofloxacin should therefore be used with caution in patients with central nervous system disorders, as such patients may be predisposed to seizures. Increased and decreased serum levels of phenytoin have been reported in patients treated with concomitant ciprofloxacin. Intravenous ciprofloxacin have been reported to result in a decrease in phenytoin levels. ${ }^{(2-6)}$ Although the exact mechanism of such an interaction is unknown, several possible mechanisms have been proposed - ciprofloxacin may decrease phenytoin concentrations by: (a) inducing phenytoin metabolism; (2) (b) disrupting the enterohepatic circulation of phenytoin; ${ }^{(3)}$ (c) shifting the volume of distribution of phenytoin; ${ }^{(6)}$ or $(d)$ increasing the renal excretion of phenytoin by inhibiting tubular reabsorption. ${ }^{(5)}$

Oral ciprofloxacin has been reported to result in increased phenytoin levels in an elderly female patient with Alzheimer's disease and hypertension, when used in combination with phenytoin..$^{(7)}$ In a pharmacokinetic study involving four healthy participants, no significant changes in phenytoin levels were demonstrated when oral ciprofloxacin was given concomitantly. ${ }^{(8)}$ Another small study of seven epileptic patients concluded that concomitant oral ciprofloxacin and phenytoin resulted in a modest $24 \%$ increase in phenytoin levels, although no clinical changes in phenytoin efficacy were reported. ${ }^{(9)}$
As ciprofloxacin may decrease serum phenytoin levels or increase seizure frequency, phenytoin plasma levels and seizure frequency should be monitored closely when ciprofloxacin is added to or discontinued from therapy. Phenytoin dose should be adjusted as needed, based on phenytoin levels and symptoms of seizure activity. When ciprofloxacin is discontinued from therapy, readjustment of phenytoin doses may be required. Additional studies that examine the effects of different administration routes on the interaction between phenytoin and ciprofloxacin are needed.

\section{REFERENCES}

1. CILOXAN $^{\circledR}$ (Ciprofloxacin $\mathrm{HCl}$ ophthalmic solution). In: Alcon [online]. Available at: http://ecatalog.alcon.com/PI/CiloxanSol_us_en.pdf.

2. Dillard ML, Fink RM, Parkerson R. Ciprofloxacin-phenytoin interaction. Ann Pharmacother 1992; 26:263.

3. Brouwers PJ, de Boer LE, Guchelaar HJ. Ciprofloxacin-phenytoin interaction. Ann Pharmacother 1997; 31:498.

4. Otero MJ, Moran D, Valverde MP. Interaction between phenytoin and ciprofloxacin. Ann Pharmacother 1999; 33:251-2.

5. McLeod R, Trinkle R. Comment: unexpectedly low phenytoin concentration in a patient receiving ciprofloxacin. Ann Pharmacother 1998; 32:1110-1.

6. Pollak PT, Slayter KL. Comment: ciprofloxacin-phenytoin interaction. Ann Pharmacother 1997; 31:1549-50.

7. Hull RL. Possible phenytoin-ciprofloxacin interaction. Ann Pharmacother 1993; 27:1283.

8. Job ML, Arn SK, Strom JG, Jacobs NF, D'Souza MJ. Effect of ciprofloxacin on the pharmacokinetics of multiple-dose phenytoin serum concentrations. Ther Drug Monit 1994; 16:427-31

9. Schroeder D, Frye J, Alldredge B, Messing R, Flaherty J Jr. Effect of ciprofloxacin on serum phenytoin concentrations in epileptic patients. Pharmacotherapy $1991 ; 11: 275$. 